\title{
Tracing the $\mathrm{CO}$ "ice line" with $\mathrm{C}^{17} \mathrm{O}$ in an MRI-active protoplanetary disk around a young sun
}

\author{
Mo Yu*i \\ University of Texas at Austin \\ E-mail: moyueastro.as.utexas.edu \\ Karen Willacy \\ NASA/JPL \\ E-mail: Karen.Willacyejpl.nasa.gov

\section{Sarah Dodson-Robinson} \\ University of Texas at Austin \\ E-mail: sdreastro.as.utexas.edu
}

The properties of the midplane of planet-forming protostellar disks remain largely unprobed by observations due to the high optical depth of common molecular lines and the continuum. However, rotational emission lines from rare isotopologues may have optical depths near unity in the vertical direction, so that the lines are strong enough to be detected, yet remain transparent enough to trace the disk midplane.

Here we present a chemical model of an MRI-active protoplanetary disk including different $\mathrm{C}$ and $\mathrm{O}$ isotopes and detailed photochemical reactions. The $\mathrm{CO}$ condensation front is found to be at 1.5 AU in the disk midplane around a solar like star, and its location remains almost unchanged during $3 \mathrm{Myr}$ of evolution. The optical depth of low-order rotational lines of $\mathrm{C}^{17} \mathrm{O}$ is around unity, which suggests it may be possible to see into the disk midplane using $\mathrm{C}^{17} \mathrm{O}$.

ALMA observations of such lines would provide estimates of the disk midplane temperature if the $\mathrm{CO}$ ice lines were spatially or spectrally resolved. With our computed $\mathrm{C}^{17} \mathrm{O} / \mathrm{H}_{2}$ abundance ratio, one would also be able to measure the disk masses by measuring the intensity of gas emission.

Frank N. Bash Symposium 2013: New Horizons in Astronomy

October 6-8, 2013

Austin, Texas

\footnotetext{
* Speaker.

${ }^{\dagger}$ This work was funded by NASA grant NNX10AH28G to SDR and KW. KWs work was conducted at the Jet Propulsion Laboratory, California Institute of Technology, under contract with the NASA Aeronautics and Space Administration.
} 


\section{Introduction and Methods}

The temperature, density, and composition of protoplanetary disks determine the materials available for planet formation. However, fundamental properties of protostellar disks such as midplane temperature, turbulent viscosity, and disk mass are currently poorly constrained by observations. Here we seek a molecule that is optically thin enough to probe the physical conditions of protostellar disk midplanes yet still remain observable with sub-mm telescope arrays.

We construct chemical evolution models of T-Tauri disks including different $\mathrm{C}$ and $\mathrm{O}$ isotopes. The model is built upon Magnetorotational Instability - active disks (hereafter MRI, Balbus \& Hawley 1991) from Landry et al. (2013), which include a viscosity prescription for accretion driven by MRI turbulence. We built the chemical model based on the UMIST database RATE95 (Millar et al.1997). The model includes gas phase reactions, grain surface reactions, freeze out, thermal desorptions, and various photochemical reactions. The computational method is similar to that of Dodson-Robinson et al. (2009).

\section{Results and Summary}

One significant feature of MRI-active disk models is the formation of a "dead zone" (Gammie et al. 1996), where the MRI turbulence is shut down due to insufficient ionization on the disk midplane. The lack of viscous heating in the dead zone yields a cold midplane and a hot disk surface. We found the dead zone to be between $2 \mathrm{AU}$ and $16 \mathrm{AU}$ on the disk midplane, leading the $\mathrm{CO}$ condensation front to be at $1.5 \mathrm{AU}$ on the disk midplane from the central star, and its location remains almost unchanged during $3 \mathrm{Myr}$ of evolution. The $\mathrm{CO}$ abundance is found to be high again at the end of dead zone at $15 \mathrm{AU}$, forming an"outer ice line", beyond which CO ice evaporates due to the viscous heating.

As the disk evolves, $\mathrm{CO}$ gradually reacts to form hydrocarbons then freezes on grains in the disk midplane, result in a large decrease in the $\mathrm{CO}$ abundance. The reaction chain is mainly triggered by helium ionized by cosmic ray particles. Then the ionized He reacts with $\mathrm{CO}$ to produce $\mathrm{C}^{+}$.

The ion-exchange reaction between $\mathrm{C}$ and ${ }^{13} \mathrm{C}$ dominates in determining the carbon isotopic ratio in $\mathrm{CO}$ :

$$
{ }^{13} \mathrm{C}^{+}+\mathrm{CO} \leftrightarrow{ }^{13} \mathrm{CO}+\mathrm{C}^{+}+35 \mathrm{~K}
$$

Since the forward reaction is energy-favorable, the $\mathrm{C}^{+}$to ${ }^{13} \mathrm{C}^{+}$ratio becomes higher than the cosmic abundance ratio. Reaction $\mathrm{C}^{+}+\mathrm{H}_{2} \rightarrow \mathrm{CH}_{2}^{+}+$photon can be a few hundred times more efficient for $\mathrm{C}^{+}$than for ${ }^{13} \mathrm{C}^{+}$, resulting in a low $\mathrm{C} /{ }^{13} \mathrm{C}$ ratio in $\mathrm{CO}$, and a high $\mathrm{C} /{ }^{13} \mathrm{C}$ ratio in hydrocarbons. The time evolution of the $\mathrm{CO}$ to ${ }^{13} \mathrm{CO}$ ratio on the disk midplane is shown in Figure 1. Due to the small reaction rate of cosmic ray ionizations, the reaction network doesn't reach an equilibrium until about $1 \mathrm{Myr}$.

We estimate the optical depth at each radius by integrating the absorption efficiency over the disk height. The optical depth of low-order rotational lines of $\mathrm{C}^{17} \mathrm{O}$ is around unity, which suggests it may be possible to see into the disk midplane using $\mathrm{C}^{17} \mathrm{O}$. The optical depth of different $\mathrm{CO}$ isotopologues in the $\mathrm{J}=3-2$ line is shown in Figure 2. Different isotopologues of $\mathrm{CO}$ trace different 



Figure 1: Left: Isotopic ratio of CO istopologues on the disk midplane. Right: Optical depth of CO 3-2 lines (time evolution) in the MRI disk $\left(0.015 M_{\odot}\right)$.

layers of the disk due to their different abundances. Observing more than one of them simultaneously can provide information of the vertical structure, and better constrain the disk model.

Ice condensation fronts can be identified in spatially resolved molecular line maps by the drop in gas emission intensity caused by freezeout of the molecule (see Qi et al. 2011, and 2013), or by the absence of low velocity components in a resolved spectral line. ALMA observations of this line would provide estimations of the disk midplane temperature if the $\mathrm{CO}$ ice lines were spatially or spectrally resolved. Additionally, with our computed $\mathrm{C}^{17} \mathrm{O} / \mathrm{H}_{2}$ abundance ratio, observers would be able to measure the disk masses by measuring the intensity of gas emission.

\section{References}

[1] Balbus \& Hawley, 1991, ApJ, 376, 214

[2] Bergin et al. 2013, Nature, 493, 644

[3] D’Alessio et al. 2006, ApJ, 638, 314

[4] Gammie et al. 1996, AJ, 457, 355G

[5] Landry et al. 2013, ApJ, 771, 80

[6] Shakura \& Sunyaev, 1973, A\&A, 24, 337

[7] Qi et al. 2011, ApJ, 740, 84

[8] Qi et al. 2013, Science, 341, 630Q 